\title{
PLC Control of Vending Machine and Design of its Simulation System ZHOU Zhengjie ${ }^{1, a}$ \\ ${ }^{1}$ Guangxi Technological College of Machinery and Electricity, Nanning city, Guangxi Prov. China 530007 \\ azzj0502@163.com
}

Keywords: Vending machine, PLC software control, data communication

\begin{abstract}
Along with the social civilization progress and the development of advanced science and technology, automatic vending machines are widely used in bus station, shopping malls, hospitals, communities, schools and other public places, which brings great convenience to people's travel and life, such as all kinds of forming small packaging commodity and beverage, tickets, card, card and etc. With the speeding up the pace of life and the prosperity of market economy, as a representative of the automatic service facilities, the vending machine will be more and more widely used.
\end{abstract}

\section{The main functions and hardware constructions of vending machine}

Vending machine is so-called the 24-hour miniature supermarkets, which is separated throughout the densely populated places. With the development of urban construction as well as the infinite market potential, vending machine is used in indoor and outdoor to facilitate pedestrian access, so it should have characteristics of environmental adaptation ability, high reliability and strong anti-interference. PLC is the controller in response to the bad industrial environment, so most of the vending machine set the PLC as the control core parts. Because the vending machine has the functions of monetary transmission, currency recognition, amount of calculation, goods, work and other functions, it is a comprehensive control system. The main work process of the vending machine and functions are as follows

(1) Money is put from slot; photoelectric sensor acquires currency data information, obtains the value and judges the authenticity.

(2) Then it passes obtained information to communication module, PLC control system accepts message communication module, main control system shows the value, and then starts the panel buttons showing what aisle is in stock and which aisle has been sold out, waiting for the customer to choose commodities through the keys.

(3) The vending machine automatically sends goods out, and then waits for customers to take the chosen goods.

(4) Then vending machine shows the RMB balance, if the balance is enough, customers can choose to a new product or to get change; if it is insufficient or customers don't want to buy other goods, they can delay work.

(5) The system resets and completes sales.

(6) When selected goods and input inadequate or identifying counterfeit money, the system will start alarm procedures.

\section{The construction components of the vending machine}

Product showcase. Product showcase consists of the transparent window of organic board, goods shelves, commodity price tags, buttons and lights of choose and buy, which is put in the inside of the door to open the door, opening the door to replace the types of commodities and commodity prices.

Goods driving mechanism. Vending machines can put a variety of goods, including beverages, food, daily necessities and so on. We put goods to the pickup through the spiral device with motor driving.

Coin recognition part. Coin identification signal is the input signals to PLC, its function is 
extremely important. Coin recognition system consists of three parts: coin identification device, coin classified storage device and automatic coin change device.

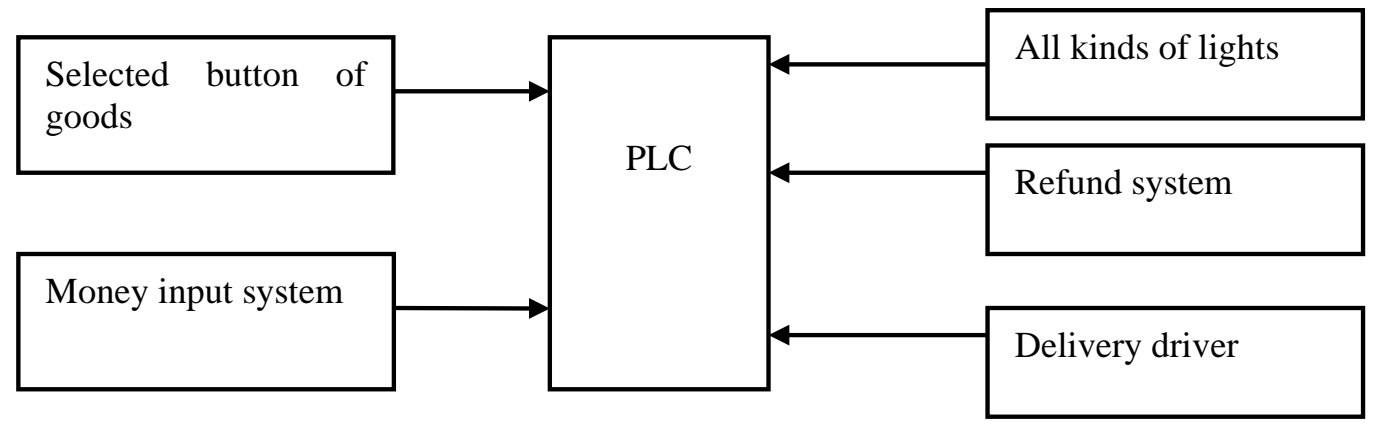

Chart 1: the hardware structure of control system

PLC control system is the core part of the vending machine control part, which controls the vending machine to identify coin, get the coin classified stored, automatic display input amount, automatically remove the purchase of goods and refund money change, and other functions in the process of the vending. At the same time, the system also adds a preset price, goods automatic counting and automatic check fault alarm functions, equivalent to the "brain" of the vending machine. The hardware structure diagram of control system is as shown in chart 1.

\section{The design of PLC control system}

PLC uses CPU as the core, a general industrial automatic control device, a combination of computer technology and communication technology, which is one of the three pillars of modern industrial control (PLC, robots and CAD/CAM). Working principle of PLC is consistent to the working principle of the basic computer, which can be simply stated as under the system application, to fulfill the users' commission through running the application. But PLC also has its own characteristics. PLC becomes a kind of special machine after loaded special program in determining the tasks and it adopts circular scanning method, work task management system and the application execution to complete circular scanning mode.

Lower machine control core component PLC chooses Omron CPM2A - 60 CDR - A model, the simulation work vending machine control process is shown in chart 2 . The vending machine PLC ladder diagram is divided into the following 8 parts: internal transfer price in the early years of operation process, coin-operated process, price comparison, choose commodities, commodity sales record process, work process, complementary goods and commodities sales statistics. PLC program execution needs to add and subtract, compare the arithmetic and logical operations etc.

Simulation images designed according to requirements of the system function mainly includes the "vending machine simulation trade screen", "out of stock items added scenes" and "sales reports". The later two screen content that is associated with the first picture content, the picture can jump to each other through the switch button. The pixels of the image uses king view toolbox, galleries and paste a bitmap way, including dynamic graph element with variable in the database system, the custom I/O or memory variables to establish corresponding relationship, only can be modified according to the requirement of the animation connection, see in table 1.

Table 1

\begin{tabular}{|l|l|l|l|l|l|}
\hline Input the signals & Output the signals \\
\hline Name & Code & Input number & Name & Code & Output number \\
\hline 5yuan entrance & & X1 & Soda signal & EL & V0 \\
\hline 10yuan entrance & & X2 & Coffee signal & EL & V1 \\
\hline 20yuan entrance & & X3 & Balance insufficient & EL & Y2 \\
\hline Soda button & SB1 & X10 & Soda entrance & KM1 & Y3 \\
\hline Coffee button & SB2 & X11 & Coffee entrance & KM2 & Y4 \\
\hline No buying refund button & SB3 & X20 & Refund signal & EL & Y5 \\
\hline & & & Refund entrance & KM3 & Y7 \\
\hline
\end{tabular}




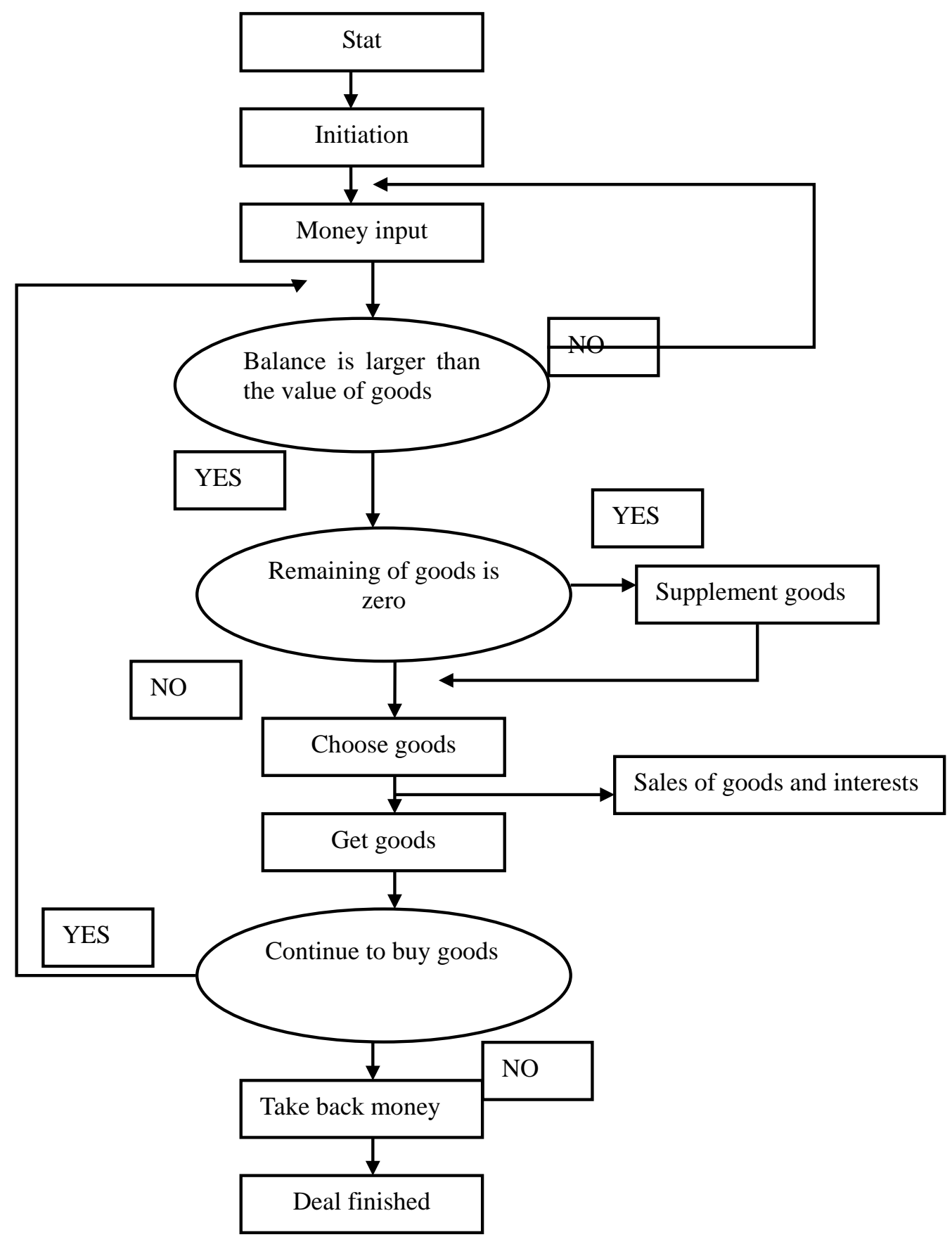

Chart 2: the control flow chart of simulation vending machine

\section{Database custom variables}

Images of dynamic figure element changes over king view database variables which includes custom with PLC I/O variables corresponding to the register address. In addition, due to the needs of the simulation, it also has nothing to do with the PLC memory variables, such as "coin".

\section{Screen design of vending machine simulation trade}

Virtual vending machine state simulation trade screen designed according to the vending machine control requirements and process, images are divided into insert coin area, amount and settlement area, the choosing and buying goods area, pickup area and work area. The simulation system completes one transaction for coin, select, click the picture in order to take the goods and refund money and draw money button, which is consistent with the actual vending machine process.

The initial run screen status is as follows: the amount of settlement area data format for three integers and 1 decimal places, at this point is shown as 0 ; each commodity "residual" number of 10 , "the price" display format for one integer 2 decimal places.10 Yuan currencies such as the graphics, "shortage" alarm light, "select" button, pickup mouth commodity graphics are hidden, will not buy 
anything at this time.

\section{Design of goods supplement frame}

Shortage of goods supplement the picture simulates alarm and supplements the shortage of goods. When the "surplus" a goods corresponding numerical display to 0 , in figure 3 the goods from the corresponding alarm indicator lights flashing and, prompt the goods is sales, in a state of out of stock, needs to be supplement. If you click the lights beside the corresponding button ("01 goods supplement" button), then send a signal ON, the input to the PLC, PLC program execution results for 01 the data register, the number of goods to buy the commodity sales of 001 , and the number of "surplus" buy goods area corresponding display into 10, the shortage of the goods at the same time alarm light is hidden, and thus realize the supplement goods.

\section{Sales statistics}

Commodity sales statistics as shown in table 2, it calls the king view embedded type create report system, using the report function real-time record all kinds of merchandise sales and the sales volume of all commodities in order to master the goods sales. The report displays the number of sales in a closed king view software after the restart still maintaining the original state, only in the click record "reset" button, it makes the corresponding register contents in PLC, picture report "value" column of the reset to zero value.

Table 2

\begin{tabular}{|l|l|}
\hline \multicolumn{2}{|c|}{ Sales and sales amount statistics of goods } \\
\hline Kinds of goods & Quantity \\
\hline 01sales amount of soda(unit) & 1 \\
\hline 02 sales amount of coffee (unit) & 2 \\
\hline 03 sales amount of cigarette (unit) & 0 \\
\hline 04 sales amount orange drinks(unit) & 5 \\
\hline Total sales amount(yuan) & 17.5 \\
\hline
\end{tabular}

\section{Dynamic figure instructions}

Images of dynamic figure element of animation connection require for the expression or command language sometimes to consider the design of the PLC program, such as the configuration screen the corresponding commodity "residual" numerical range is $10 \sim 0$, it increases with the increasing number click the "select" button (that is, the increase of the sales of the commodity) and decreasing, while the PLC program execution to plus 1 operation, therefore, king view animation connection "analog output value" expression should be set to 10-01 "sales"

\section{Summary}

Simulation system has been debugging and running successfully, it has been applied to PLC teaching and achieved good teaching effect, achieved the expected teaching objectives. The process of the simulation system matches the actual trading process of vending machines, and on the operation and management concept of dual, it realizes the function of a more perfect vending machine simulation system with certain practical value.

\section{References}

[1] Zhang Wanzhong, Applied technology of PLC[M]. Beijing: Chemical Industry Press,2002.

[2] Zhu Saiping, PLC application technology[M]. Beijing, China labor social security publishing house,2006.

[3] Zhao Yuhua, Design of PLC virtual simulation system based on configuration technology[J]. Application technology,2005(12):48-50.

[4] Zhao Hongmei, Zhang Jun, PLC control system of vending machine[J].Technology press, 2009(3):37-39.

[5] Sun Zhaoqiang, The principle and application of programmable controller tutorial[M].Beijing: Tsinghua University Press,2008. 